\title{
Balkanologie
}

Balkanologie Revue d'études pluridisciplinaires

Vol. VIII, $n^{\circ} 2$ | 2004

Volume VIII Numéro 2

\section{Bertrand (Gilles), Le Conflit Hélléno-Turc}

Paris : Maisoneuve et Larose, 2003, 390 pages

Joseph Krulic

\section{CpenEdition}

Journals

Édition électronique

URL : http://journals.openedition.org/balkanologie/2051

DOI : 10.4000/balkanologie.2051

ISSN : 1965-0582

Éditeur

Association française d'études sur les Balkans (Afebalk)

Édition imprimée

Date de publication : 1 décembre 2004

ISSN : 1279-7952

Référence électronique

Joseph Krulic, «Bertrand (Gilles), Le Conflit Hélléno-Turc », Balkanologie [En ligne], Vol. VIII, n² 2 | 2004,

mis en ligne le 20 janvier 2010, consulté le 17 décembre 2020. URL : http://journals.openedition.org/

balkanologie/2051 ; DOI : https://doi.org/10.4000/balkanologie.2051

Ce document a été généré automatiquement le 17 décembre 2020.

(c) Tous droits réservés 


\section{Bertrand (Gilles), Le Conflit Hélléno- Turc}

Paris : Maisoneuve et Larose, 2003, 390 pages

Joseph Krulic

\section{RÉFÉRENCE}

Bertrand (Gilles), Le Conflit Hélléno-Turc, Paris : Maisoneuve et Larose, 2003, 390 pages

1 L'ouvrage de Gilles Bertrand, transposition d'une thèse soutenue sous la direction de Bertrand Badie, constituera un ouvrage de référence sur une question complexe qui suscite bien des passions. Le constat de base est que depuis longtemps, et de manière accentuée depuis l'invasion par l'armée turque en 1974, les relations entre la Grèce et la Turquie sont tendues. S'agit-il, à titre unique ou principal, d'un conflit politicostratégique inter-étatique classique, d'un écho d'un "clash de civilisations »? Ces questions sont posées par l'auteur qui y répond négativement. Il se fixe pour objectif de dépasser cette approche traditionnelle. Il veut se situer dans une perspective sociohistorique et vise à mettre en évidence les mutations intervenues dans le conflit après la fin de la guerre froide, à la lumière du contexte international et du problème kurde.

2 Toutefois, l'analyse qu'il fait des multiples facteurs du conflit inter-étatique constituent son apport le plus durable. L'auteur connaît admirablement plusieurs domaines du droit international, notamment le droit de la mer et le droit aérien. Cela lui permet d'approfondir l'explication des nombreux contentieux ou crises, la délimitation des frontières, des eaux territoriales ou des couloirs aériens, cause, accélérateur ou révélateur de nombreuses crises bilatérales entre les deux pays. Il évite les généralités et les approximations. Les tableaux très précis sur la course aux armements entre les deux pays, sur leurs évolutions économiques du point de vue macro-économique, les analyses de la vie politique interne de la Grèce et de la Turquie en relation avec les enjeux internationaux constituent une actualisation et une synthèse précieuse de 
données dispersées dans les revues, les sources officielles et les thèses de doctorat de droit, de science politique, d'histoire.

3 L'auteur consacre de nombreuses analyses au conflit chypriote. Il se livre à de stimulantes comparaisons avec la division irlandaise, la coupure Inde-Pakistan, IsraëlPalestine, qui constituent tous, comme il le note, des héritages coloniaux britanniques. Il existerait ainsi un véritable modèle de décolonisation/partition britannique et postbritannique. À cet égard, l'apport de son livre sur ce sujet très étudié nous semble se situer dans l'analyse des négociations d'adhésion et de pré-adhésion de Chypre et de la Turquie à l'Union européenne. Dans le chapitre «l'Union européenne à l'épreuve du conflit helléno-turc», il montre l'embarras des structures de Bruxelles sur le sujet, comme les contradictions des pays-membres, dont le rôle apparaît singulièrement limité, à l'exception très relative du Royaume-Uni.

Certains points de vue pourraient être discutés. L'auteur, comme la majorité des politologues spécialistes de relations internationales, est très hostile à l'État-nation comme « machine à fabriquer des ennemis » (conclusion, p. 351). Il estime, à propos de la rencontre entre İsmet İnönü et Charles De Gaulle, en 1964 que l'entretien « montre surtout un De Gaulle enfermé dans la logique des États-nations et celle de la politique des puissances » (p. 219). Dans l'étude du politique, Gilles Bertrand ne montre qu'un goût modéré pour la «compréhension" (Verstehen) au sens épistémologique des sociologues ou philosophes allemands de la grande époque des années 1885-1930, de Tönnies à Karl Jaspers en passant par Max Weber et Edouard Dilthey, des acteurs ou des auteurs qu'il récuse. Max Weber (cité, notamment p. 175 sur les «hommes charismatiques») considérait que la "guerre des dieux» (entre les valeurs, le choix étant largement indécidable selon Weber) ne s'achèverait jamais. On pourrait, dire, glissant un trait d'humour sur un ouvrage très sérieux, que Gilles Bertrand, après bien d'autres, conformément à la vision du monde des chercheurs "internationalistes ", pense que l'internationalisme post-communiste fera le genre humain, à Athènes, comme à Istanbul, qu'il prenne la forme de l'universalisme "habermassien » ou de la « juste mémoire » de Paul Ricœur : ce dernier étant cité dans la conclusion. Huntington est sa " tête de Turc " prévisible (à chacun son Turc!). Mais pour réfuter Huntington, figure de rhétorique obligatoire dans les sciences sociales françaises, il ne fait pas appel à quelqu'un comme Fernand Braudel, historien de la très longue durée géographique et économique, non cité à notre connaissance. Pour se situer dans une perspective sociohistorique, il évacue de son champ de vision la longue durée socio-historique, tout en tenant compte (voir supra) de la macro-économie la plus récente : son champ de vision, largement politique, tient compte de la guerre d'indépendance grecque après 1823 , n'omet pas de signaler les massacres, expulsions et «échanges de population » entre Grèce et Turquie depuis 1829 (voir, par exemple, le tableau, p. 133, de la disparition progressive de la minorité grecque-orthodoxe de Turquie).

Gilles Bertrand analyse les rapprochements entre la Grèce et la Turquie. Il prend bien soin de distinguer le « rapprochement » de la « réconciliation » (p. 273), dont le modèle serait la réconciliation franco-allemande. Cette approche rigoureuse mérite d'être saluée. Ces rapprochements ont des précédents en 1930-1931 (voir pp. 173-177), où İsmet Inönü et le ministre des Affaires étrangères turc, Rüstü Aras, sont reçus triomphalement à Athènes. La plupart des initiatives sont d'origine politique depuis 1987. La classe politique grecque, par exemple, est beaucoup plus favorable qu'on le croit à la Turquie ou, en tout cas, à son adhésion conditionnelle à l'Union européenne 
(UE). L'appartenance commune à l'OTAN, comme l'adhésion de la Grèce à l'UE en 1981, limite le risque de guerre ouverte, tout en excitant, selon une hypothèse fréquente des spécialistes, les micro-conflits et les rivalités. Dans une troisième partie, suivant en cela une longue tradition de spécialistes de relations internationales, il n'omet pas de faire l'inventaire des initiatives de paix non étatiques. Les «sociétés civiles" grecques et turques (chapitre 7) et les initiatives en faveur d'une « réconciliation » à Chypre, depuis les universitaires, patrons, ordres professionnels, montrent que des acteurs nonétatiques se sont souvent efforcés de rapprocher les deux (ou trois, avec Chypre) sociétés. Le bilan est certes contrasté (pp. 304-305), mais le tableau montre qu'il ne s'agit pas d'un affrontement sans nuance. En cela d'ailleurs, par l'analyse précise de réalités identifiables, plus que dans ses pages de réfutation théorique contre Huntington, Gilles Bertrand réfute, par l'exemple, le schéma de la «guerre de civilisations ».

6 À plusieurs reprises (conclusion, p. 353, mais aussi les pp. 183-192 traitant des « guerres yougoslaves et risques de régionalisation du conflit hélléno-turc »), l'auteur analyse le comportement de la Grèce dans les conflits yougoslaves. Il montre, de manière convaincante, que la Grèce fut moins pro-serbe qu'on ne l'a dit en Europe occidentale. Les acteurs " yougoslaves » comme Slobodan Milošević et Radovan Karadžić ont, certes, joué sur la sympathie qu'inspirait leur cause "aux ultranationalistes grecs » mais, passant de l'analyse au jugement de valeur, au risque de trop prouver, il soutient que le partage de Dayton « reproduit les pires erreurs de la diplomatie fondée sur la puissance et la croyance que les chefs, individus rationnels agissant suivant les préceptes de la Realpolitik, peuvent conclure une paix solide». On l'aura compris, l'auteur n'appartient pas à l'école "réaliste ». Le komšiluk de Bosnie lui semble constituer la réfutation évidente d'Huntington: l'affrontement inéluctable entre chrétiens orthodoxes et musulmans ne résiste pas à l'épreuve de la réalité, c'est-à-dire de la coexistence et du «bon voisinage » (komšiluk en Bosnie-Herzégovine, avec une note sur le livre de Xavier Bougarel, présenté comme source de cette évidence, p. 184). Sa lecture de Xavier Bougarel nous semble rapide et trop peu nuancée. Le komšiluk serait-il vraiment cette fraternité post-moderne, " habermassienne » avant la lettre (L'intégration républicaine, d'Habermas, est cité p. 353, dans la conclusion), résistant à toutes les conjonctures politiques ? Il en est de même de sa lecture de Paul Ricœur. Une chose est de rappeler la «belle expression » de « juste mémoire » (p. 355) ; mais, outre que la question est fort complexe - comme le montre d'ailleurs le livre de Paul Ricœur auquel il est fait référence comme aux débats qu'il a suscités ${ }^{1}$ - Paul Ricœur lui-même, philosophe féru de théologie protestante dont les analyses sur cette question se prolongent depuis plus de 60 ans, n'a jamais négligé les aspects religieux et culturels des conflits humains ${ }^{2}$, notamment dans le cas du conflit entre Israël et les Palestiniens, mais chez lui, l'herméneutique des anciennes traditions permet d'alimenter de nouvelles sources de conflits ou, au contraire, de pacifier la mémoire par de nouvelles interprétations. Gilles Bertrand, reprenant les recherches de tous ordres des sciences humaines, a raison de souligner (p. 19: " nation et ethnicité : deux concepts problématiques ») le caractère fabriqué et récent des nationalismes; mais, de ce caractère récent, faut-il conclure, même implicitement, à la facile désinvention de ces traditions récentes? Paul Garde, dans un livre lui-même récent ${ }^{3}$, tout en partageant, en pratique, de nombreuses conclusions de Gilles Bertrand, récuse l'hostilité sans réserve de ce dernier envers les États-nations et conclue « exorciser, par une analyse objective, les illusions forgées par les nationalismes, et dénoncer les horreurs qui en découlent, ne doit pas conduire à 
sous-estimer les identités nationales, réalité profonde qui jouera nécessairement un rôle dans les états démocratiques à construire ou à consolider dans cette partie de l'Europe ». On pourrait faire de semblables remarques sur le débat entre « réalistes » et « internationalistes » dans l'analyse des relations internationales ou de la société. Il est, certes, possible de préférer Bertrand Badie à Raymond Aron ou Henry Kissinger, Jürgen Habermas à Max Weber ${ }^{1}$. Mais c'est une chose distincte que de croire ce débat déjà tranché, des points de vue épistémologique comme politique, lesquels ne se recouvrent pas nécessairement comme Gilles Bertrand, brouillant, certes implicitement, les frontières du politique et du savant, semble parfois l'admettre. En réalité, le débat ne peut que continuer².

7 Le livre de Gilles Bertrand, livre de références et de réflexions, renvoie ainsi son lecteur à un monde et à plusieurs mondes de réflexions possibles. En réalité, au regard de la richesse du livre, de sa pluridisciplinarité, de la polysémie des concepts qu'il utilise, il est difficile de rendre justice à l'ouvrage de Gilles Bertrand.

\section{NOTES}

1. Ricoeur (Paul), La mémoire, l'histoire, l'oubli, Paris : Seuil, 2002 ; pour un commentaire, voir Robin (Régine), «Une juste mémoire, est ce possible?», in Devoir de mémoire, droit à l'oubli, Bruxelles : Complexe, 2002.

2. Voir le recueil de textes de Lindenberg (Daniel), Maïla (Joseph), Le conflit israélo-palestinien, Paris : Desclée de Brower, 2001.

3. Garde (Paul), le discours balkanique. Des mots et des hommes. Paris : Fayard, 2004.

1. Pour un confrontation, voir Sintomer (Yves), La démocratie impossible? Politique et modernité chez Weber et Habermas, Paris : La Découverte, 1999.

2. Ibid. 